\title{
Improving impulsivity assessment using movement recognition: A pilot study
}

\author{
David Delgado-Gómez ${ }^{1}$ - Carlos Carmona-Vázquez ${ }^{2}$ - Sofia Bayona ${ }^{3}$. \\ Juan Ardoy-Cuadros ${ }^{4}$ - David Aguado ${ }^{5}$ Enrique Baca-García ${ }^{6}$. \\ Jorge Lopez-Castroman ${ }^{7}$
}

Published online: 20 October 2015

(C) Psychonomic Society, Inc. 2015

\begin{abstract}
The Continuous Performance Test (CPT) is a widely used computerized test to assess impulsivity. This article proposes the use of a CPT variant based on movement recognition to obtain more accurate measurements of impulsivity. In this pilot study, 22 volunteers participated in a CPT experiment responding to the stimuli by raising his or her dominant hand instead of pressing the space bar in a keyboard. Using this method, correlations of self-reported impulsivity with number of commission errors and average reaction time improved those obtained with standard CPT.
\end{abstract}

Keywords Continuous Performance Tests $\cdot$ Kinect . Impulsivity $\cdot$ Barratt's Impulsiveness Scale

Electronic supplementary material The online version of this article (doi:10.3758/s13428-015-0668-y) contains supplementary material, which is available to authorized users.

David Delgado-Gómez

david.delgado@uc3m.es

1 Department of Statistics, Universidad Carlos III, c/Madrid, 126, 28903 Getafe, Spain

2 Faculty of Telecommunications, Universidad Carlos III, Leganés, Spain

3 Department of Computer Science, Universidad Rey Juan Carlos, Móstoles, Spain

4 Departamento de Medicina y Cirugía, Psicología, Medicina Preventiva y Salud Pública, Universidad Rey Juan Carlos, Alcorcón, Spain

5 Knowledge Engineering Institute, Autonomous University of Madrid, Madrid, Spain

6 Department of Psychiatry, Fundación Jimenez Diaz Hospital, Madrid, Spain

7 Department of Adult Psychiatry, CHRU Nimes and INSERM U1061, Nimes, France

\section{Introduction}

Although a consensual definition does not exist, the multifaceted construct of impulsivity can be understood as "a predisposition toward rapid, unplanned reactions to internal or external stimuli without regard to the negative consequences of these reactions to the impulsive individual or to others" (Moeller, Barratt, Dougherty, Schmitz, \& Swann, 2001). Impulsivity appears in every major system of personality (Whiteside \& Lynam, 2000), characterizes several mental disorders - such as drug use disorders or borderline personality disorder (Chamberlain \& Sahakian, 2007), and has been directly associated with educational failure (Spinella \& Miley, 2003). Of note, several dimensions of impulsivity, such as behavioral disinhibition, risky decision-making, or delay discounting, have been described (Avila, Cuenca, Félix, Parcet, \& Miranda, 2004; Dom, De Wilde, Hulstijn, \& Sabbe, 2006; Glicksohn, Leshem, \& Aharoni, 2005). Thus, impulsivity traits can be defined and measured in different ways. In this study, we will use the term impulsivity to mean behavioral disinhibition, which refers to acting without thinking or failing to inhibit an initiated response (Iacono, Malone, \& McGue, 2007).

The assessment of an individual's impulsivity is frequently carried out through questionnaires in clinical settings (Chamberlain \& Sahakian, 2007). However, this approach presents some weaknesses. Firstly, the construction of a questionnaire is influenced by culture and the correspondence with the actual trait is difficult to validate (Möller, 2009). Secondly, the reliability of the individual answering the questionnaire is not guaranteed and might be affected by life events. For instance, psychiatric patients may modify their answers in order to get access to drugs or to be discharged (Marshall et al., 2010). Thirdly, questionnaires are unsuitable for repeated use because of learning biases (Chamberlain \& Sahakian, 2007). 
Thus, task-oriented computerized tests have been developed during recent decades to obtain more reliable impulsivity measures.

The Continuous Performance Test (CPT) is allegedly the most popular clinic-based measurement of response inhibition (for a review see Riccio, Reynolds, \& Lowe, 2004). CPTs consist generally of a fast presentation of continuously changing stimuli, among which an infrequent "target" requires a particular action, such as pressing a space bar (Conners \& Staff, 2000). This test allows quantifying two variables that are related to impulsivity: the average hit reaction time (HRT) and the number of commission errors. Additionally, the recent emergence of affordable motion-sensing devices into the market has opened promising lines of research that try to improve psychometric assessments using new behavioral indexes. Movement recognition using depth/color images can help to study behavioral patterns (Liu \& Shao, 2013) or to estimate attention levels combining body posture and head orientation (Stanley, 2013).

This article aims at improving the assessment of impulsivity through the CPT task using a motion sensor device (Microsoft Kinect ${ }^{\odot}$ ), which is readily available for consumer entertainment. The movement recognition ability of this kind of device captures behavioral patterns that are unnoticed by usual CPTs, such as uncompleted actions in which the subject starts the movement but does not press the space bar. We hypothesize that capturing these behaviors will provide a more accurate evaluation of impulsivity.

\section{Material and methods}

\section{Sample and set-up}

In order to evaluate the proposed method, a sample of 22 university students conducted the CPT twice, once responding through a keyboard (standard CPT) and once raising their dominant hand in front of a Kinect sensor (Kinect CPT, setup shown in Fig. 1S). Two screening questions were made to rule out psychiatric diagnoses among the participants: "Have you ever been diagnosed with a mental illness?" and "Have you ever received psychological or psychiatric treatment?" All participants responded negatively to both questions. They were also first-time users of Kinect and not aware of the study procedure. To prevent recall biases in the second test, two versions of CPT were randomly generated using the Psychology Experiment Building Language (Mueller \& Piper, 2014). The assignation of these two tests was counterbalanced and alternated for each subsequent subject, half of the participants $(\mathrm{n}=11)$ completed the keyboard version first and the other half completed the Kinect version first. The average age of the participants was 24.7 years, with a standard deviation of 4.05 . Most participants were males (63\%).

\section{Standard Continuous Performance Test (CPT)}

In this study, the CPT presented 360 letters on a computer screen with an interval of 1,2 , or $4 \mathrm{~s}$ between them, altogether lasting approximately $14 \mathrm{~min}$. The participants were asked to hit the space bar on the keyboard every time a letter, other than $\mathrm{X}$, appeared on the screen. Whenever the $\mathrm{X}$ appeared, the participants were asked to refrain from hitting the space bar. For each subject, a collection of different measures was recorded: (1) the type of stimulus and its onset time, (2) whether the participant pressed the space bar or not; and (3) the elapsed time between the emergence of the stimulus and the instant when the space bar was hit. The HRTs were measured as the elapsed time between the moment when non-X letters appeared on the screen and the moment when the space bar was hit. The commission errors represented the number of times that participants hit the space bar to an X stimulus. To make tests with different numbers of $\mathrm{X}$ stimuli comparable, we used the proportion of commission errors with respect to the total number of $X$ stimuli instead of using just the number of commission errors. Thus, HRT was calculated on non-X stimuli, while commission errors were quantified on X stimuli. A very fast HRT, when combined with high commission error rates, indicates impulsivity (Riccio et al., 2004).

\section{Kinect CPT}

A standard RGB color camera, an infrared projector, an infrared sensor, and a set of four microphones compose the Microsoft Kinect sensor. The Kinect software development kit (SDK), which can be freely downloaded from Microsoft's Kinect for Windows website, allows locating 20 different body parts of the individuals. Among them, the real-time location of the dominant hand was measured according to screen coordinates and used to establish a variant of the standard CPT. The authors can provide the software developed for the present study on request.

In this version of the CPT, the participants were placed in front of a screen and presented with non-X stimuli to which they should raise their hand and X stimuli to which they should inhibit the action. This method captures events that cannot be detected using standard CPT, particularly those in which the participant starts an action but stops it before completion. The following measures were registered to quantify HRT and commission errors: the onset time and type of stimulus, and the position of the dominant hand - in screen coordinates - every $1 / 35 \mathrm{~s}$.

The HRT was calculated as the elapsed time between the appearance of the non-X stimuli and the moment when the participants started raising their hand. This point was determined by adjusting a piecewise function to the first part of the signal, using the gradient descent technique (see Fig. 2S). The time lapse between stimuli was maintained equal to the 
original values (i.e., 1, 2 and 4 s). Please see supporting information for details of the procedure.

To avoid an overestimation of response times, the HRTs for non-X stimuli was only calculated if the hand was at resting position before the next stimulus appeared in the screen (Fig. 3S). We adjusted a regression line using six sample points before and six sample points after the appearance of the stimulus. The choice of the number of sample points was based on a sensitivity analysis. The slope of this line would be close to zero if the participant started the movement from a resting position, but it would adopt a negative value if the hand were still moving down. Thus, if the slope of the regression line exceeded a certain threshold (T1), the movement started from the resting position. The value of $\mathrm{T} 1$ was determined using a supervised learning approach by checking if every instance of a small sample of reactions had started from the resting position. T1 threshold was the value that most accurately classified the previously annotated reactions. On the other hand, to avoid an underestimation of reaction times in premature movements, a second threshold (T2) was calculated using a similar procedure. We considered that the reaction started after the emergence of the stimulus when the value of the slope was smaller than T2. In summary, the valid reactions were the ones for which the estimated value of the slope was situated between T1 and T2. Another potential method to measure the reaction of each participant could have been based on the speed of the dominant hand (Studenka, Zelaznik \& Balasubramaniam, 2012).

Finally, the following Motion-based Impulsivity Index (MBI) was used to determine commission errors:

$\operatorname{MBI}(\mathrm{i})=\left(\mathrm{H}_{\mathrm{x}}-\mathrm{h}_{\mathrm{x}}\right) /\left(\mathrm{M}-\mathrm{h}_{\mathrm{x}}\right)$

where $h_{X}$ is the height (of the dominant hand) at the instant when the $i^{\text {th }}$ stimulus appears; $\mathrm{H}_{\mathrm{X}}$ is the maximum height since the onset of a $\mathrm{X}$ stimulus until the next stimulus appears; and $\mathrm{M}$ is the maximum height attained in the interval that follows the previous or the posterior stimulus (see Fig. 4S). Notice that the numerator accounts for the displacement of the hand between the appearances of stimuli $\mathrm{i}$ and $\mathrm{i}+1$; and the denominator represents the longest displacement of the hand after the previous or posterior stimulus. Consequently, this index will be close to 0 if the individual inhibits the action and close to 1 if the action is completed. Of note, the MBI avoids the limitations of using a spatial threshold, which should be adapted to the height of each participant, as well as their reaction patterns. Below, in the results section, we suggest an approach to transform the MBI into a binary value that indicates if the examinee has made a commission. The supplementary video illustrates how the Kinect CPT works.

\section{Barratt impulsiveness scale}

All participants were native Spanish speakers and completed the 11th version of the Barratt Impulsiveness Scale (BIS-11) in its Spanish version (Oquendo et al., 2001). The BIS-11 is a self-report scale, which includes 30 items such as "I plan tasks carefully" or "I am self controlled." Each item's score ranks from 1 (rarely/never) to 4 (almost always/always). A total score for BIS-11 is obtained by summing up all item scores. Participants were asked to complete the BIS-11 assessment once the CPTs were performed to avoid any bias.

We compared the performance of Kinect CPT and standard CPT calculating the correlation of BIS-11 scores with the number of commission errors and the average reaction time in both types of CPT.

\section{Results}

Figure 1 shows an example of the trajectory of the dominant hand in Kinect CPT. The X-axis indicates the time and the Yaxis represents the height of the hand in screen coordinates. The plot is displayed in dashed lines when the individual should not raise his or her hand (X stimuli); otherwise it is displayed in solid line. The onset time of each stimulus is represented with a vertical dashed line. Two longer examples and their corresponding indexes of commission errors can be observed in Figs. 5S and 6S, respectively.

The average HRT (SD) for Kinect CPT was $0.382 \mathrm{~s}(0.05)$ while the average HRT for standard CPT was $0.371 \mathrm{~s}(0.04)$. The average proportion of commission errors (SD) was $0.31(0.16)$ for Kinect CPT and 0.39 (0.20) for standard CPT. No significant difference was found between average HRT $(\mathrm{t}(42)=0.80 ; \mathrm{p}=$ 0.42 ) or average proportion of commission errors $(\mathrm{t}(42)=1.46 ; \mathrm{p}$ $=0.15)$ using Kinect CPT and standard CPT. The average BIS11 score was 66.13 (9.22). There was a high correlation in commission errors $(r=0.568)$ and HRT $(r=0.768)$ between Kinect CPT and standard CPT. Given the relatively small sample size, statistical tests do not detect significant differences in correlations when comparing standard CPT and Kinect CPT.

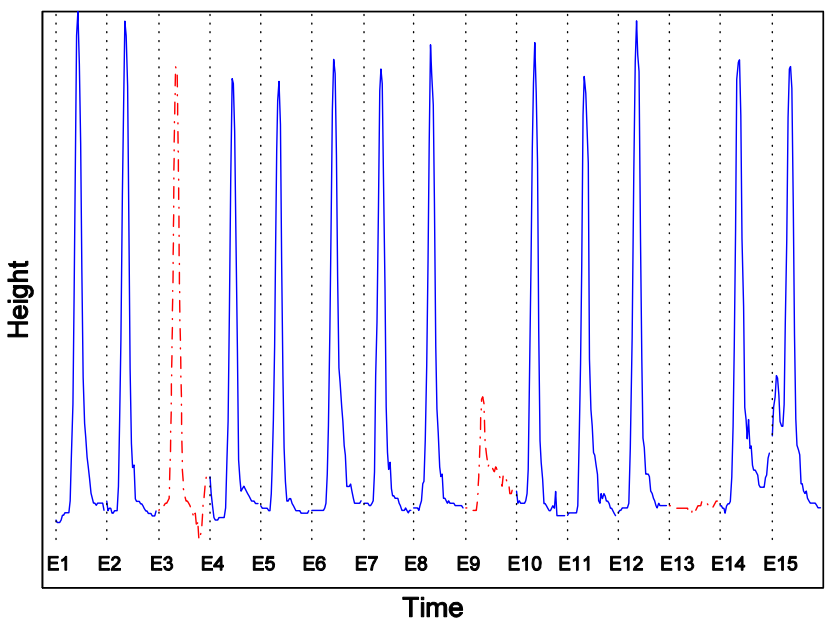

Fig. 1 Trajectory of the dominant hand for 15 stimuli. Solid lines represent the responses to non-X stimuli, while red dashed lines represent responses to $\mathrm{X}$ stimuli 
In the standard CPT task, we found a correlation between BIS-11 and commission errors of 0.217. However, for Kinect CPT this correlation depends on the threshold that identifies movements as commission errors. Figure 2 shows the correlation between commission errors and BIS-11 as a function of the selected threshold. To facilitate the comparison, a horizontal line representing the correlation obtained according to standard CPT is depicted. The highest correlation for Kinect CPT is obtained with a $6 \%$ threshold: 0.524 . This value, which more than doubles the correlation with standard CPT, clearly indicates the improvement provided by Kinect CPT despite the lack of significant differences. Indeed, regardless of the threshold value, correlations between BIS-11 and Kinect CPT are largely superior to those obtained with standard CPT. The distribution of the indexes could also be fitted to a mixture of two Gaussian distributions (Taxt, Hjort, \& Eikvil, 1990) to determine the value that best separates (statistically) both groups. Any record above this value would be considered a commission error. The intersection point of the two Gaussians defined a $13 \%$ threshold with a correlation of 0.462 .

On the other hand, the correlation between BIS-11 and HRT was -0.466 for standard CPT while this correlation was -0.534 for Kinect CPT. In both cases, the more impulsive the participant, the shorter the reaction time.

\section{Discussion}

In this article, an innovative implementation of the CPT employing the Microsoft Kinect motion sensor has been compared with a standard CPT to measure two indexes of impulsivity: commission errors and HRT. The correlation between the proportion of commission errors and the BIS-11 was

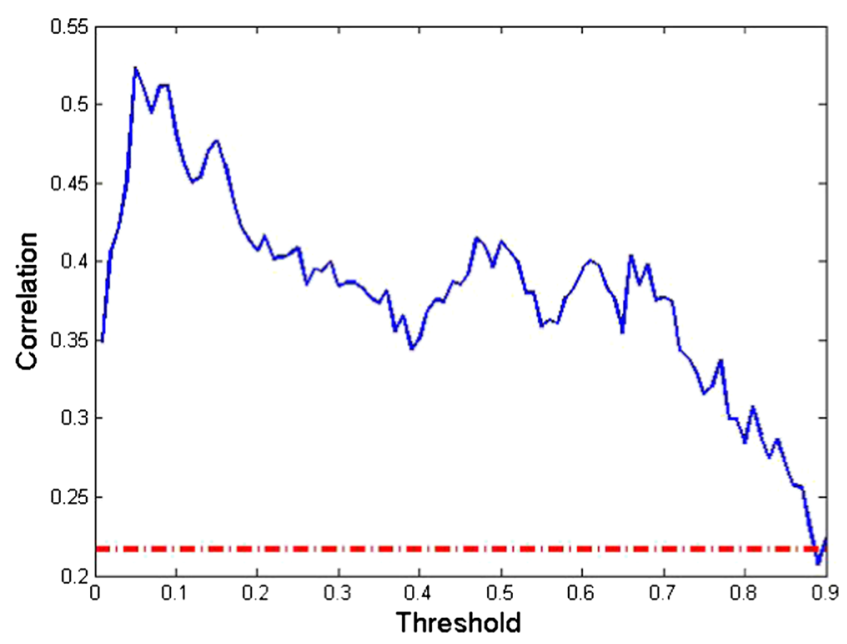

Fig. 2 Correlation between BIS-11 and the Kinect Continuous Performance Test (CPT) depending on the threshold applied to determine commission errors. The red dashed horizontal line at the bottom marks the correlation between BIS-11 and standard CPT $(\mathrm{r}=0.217)$ positive for both methods, confirming that impulsive participants make more commission errors than non-impulsive ones (Conners \& Staff, 2000). Likewise, the correlation between HRTs and BIS-11 were negative for both types of CPTs, reflecting the shorter reaction times found among impulsive individuals (Conners \& Staff, 2000). The longer distance to "hit" in Kinect CPT compared to standard CPT agrees with our finding of higher reaction times and consequently less commission errors. Participants had more time to inhibit their actions in Kinect CPT.

As hypothesized, the Kinect CPT achieved higher correlations in absolute value between both indexes of impulsivity (commission errors and HRT) and the BIS-11, than the standard CPT. These findings suggest that capturing incomplete "hits" by Kinect CPT allows a more precise measurement of impulsivity when compared with standard CPT. The relationship of impulsivity with HRT and commission errors in CPT tests is well established (Edman, Schalling, Levander, 1983). Because of the central role played by impulsivity in many psychiatric syndromes, an easy but reliable way to assess impulsivity has become an important issue in the psychiatric field. Thus, a more informative version of CPT could be an accurate and culturally unbiased measurement of impulsivity, in turn facilitating early diagnosis and personalized treatments. The utilization of a Kinect CPT would convey other potential applications derived from movement recognition: (1) patterns of movement can be combined with personality measures or other psychometrical tests to explore their interaction; (2) movement recognition analyzes non-programmed actions and thus makes easier the evaluation of handicapped or aged persons (i.e., the recognition can be adapted to the movement); (3) movement disorders could be evaluated or controlled for when using a CPT test.

The results also open new avenues for research. For instance, movement analysis of other body parts or body posture could inform about sustained attention or vigilance (Stanley, 2013). Moreover, this article has been focused on the CPT, but the development of specific tests or the adaptation of old ones could maximize the psychometric utility of motion sensor devices. As already indicated, this kind of device is increasingly affordable while in parallel specific software and algorithms for data analyses are becoming available. Thus, the implementation of movement recognition and analysis for personality assessment is now feasible in many different areas.

Some limitations should be mentioned. First, although a 1-s lapse is enough in standard CPTs to press and release the space bar, some participants found difficulties in raising and returning the hand to a resting position in the Kinect CPT version within this time interval. Second, participants became tired during the test. Analysing a shorter and horizontal movement could solve these limitations. Finally, as this is a pilot study on a small sample, we were unable to find statistical differences between correlations in standard and Kinect CPTs. 
However, the value of the correlation between BIS-11 and commission errors would be more than doubled by selecting the most performing threshold in Kinect CPT. New studies on larger and more representative samples, including clinical subjects, are necessary to confirm our findings, as well as to examine the evolution of the MBI over time.

In summary, standard and technology-enhanced measures of impulsivity on the CPT were comparable, and these measures were correlated to the participant's ratings on the BIS-11. This pilot study presents a novel method, based on movement recognition, that might increase the precision of impulsivity measures.

\section{References}

Avila, C., Cuenca, I., Félix, V., Parcet, M.-A., \& Miranda, A. (2004). Measuring impulsivity in school-aged boys and examining its relationship with ADHD and ODD ratings. Journal of Abnormal Child Psychology, 32(3), 295-304.

Chamberlain, S. R., \& Sahakian, B. J. (2007). The neuropsychiatry of impulsivity. Current Opinion in Psychiatry.

Conners, C. K., \& Staff, M. (2000). Conners' Continuous Performance Test II (CPT II V. 5). North Tonawanda.

Dom, G., De Wilde, B., Hulstijn, W., \& Sabbe, B. (2006). Dimensions of impulsive behaviour in abstinent alcoholics. Personality and Individual Differences, 42(3), 465-476. doi:10.1016/j.paid.2006. 08.007

Edman, G., Schalling, D., \& Levander, S. E. (1983). Impulsivity and speed and errors in a reaction time task: a contribution to the construct validity of the concept of impulsivity. Acta psychologica, 53, $1-8$.

Glicksohn, J., Leshem, R., \& Aharoni, R. (2005). Impulsivity and time estimation: Casting a net to catch a fish. Personality and Individual Differences, 40(2), 261-271. doi:10.1016/j.paid.2005.07.003

Iacono, W. G., Malone, S. M., \& McGue, M. (2007). Behavioral disinhibition and the development of early-onset addiction: common and specific influences. Clinical Psychology, 4, 325-348. doi:10.1146/ annurev.clinpsy.4.022007.141157
Liu, L., \& Shao, L. (2013). Learning discriminative representations from $R G B-D$ video data (pp. 1493-1500). AAAI Press.

Marshall, P., Schroeder, R., O’Brien, J., Fischer, R., Ries, A., Blesi, B., \& Barker, J. (2010). Effectiveness of symptom validity measures in identifying cognitive and behavioral symptom exaggeration in adult attention deficit hyperactivity disorder. The Clinical Neuropsychologist, 24(7), 1204-1237. doi:10.1080/13854046. 2010.514290

Moeller, F. G., Barratt, E. S., Dougherty, D. M., Schmitz, J. M., \& Swann, A. C. (2001). Psychiatric Aspects of Impulsivity. American Journal of Psychiatry, 158(11), 1783-1793. doi:10.1176/appi.ajp.158.11. 1783

Möller, H.-J. (2009). Standardised rating scales in psychiatry: methodological basis, their possibilities and limitations and descriptions of important rating scales. The World Journal of Biological Psychiatry: the Official Journal of the World Federation of Societies of Biological Psychiatry, 10(1), 6-26. doi:10.1080/15622970802264606

Mueller, S. T., \& Piper, B. J. (2014). The Psychology Experiment Building Language (PEBL) and PEBL Test Battery. Journal of Neuroscience Methods, 222, 250-259. doi:10.1016/j.jneumeth. 2013.10.024

Oquendo, M. A., Baca-Garcia, E., Graver, R., Morales, M., Montalvan, V., \& Mann, J. J. (2001). Spanish adaptation of the Barratt Impulsiveness Scale (BIS-11). European Journal of Psychiatry, 15(3), 147-155.

Riccio, C. A., Reynolds, C. R., \& Lowe, P. A. (2004). Clinical Applications of Continuous Performance Tests. Wiley.

Spinella, M., \& Miley, W. M. (2003). Impulsivity and academic achievement in college students. College Student Journal.

Stanley, D. (2013). Measuring attention using Microsoft Kinect.

Studenka, B. E., Zelaznik, H. N., \& Balasubramaniam, R. (2012). The distinction between tapping and circle drawing with and without tactile feedback: An examination of the sources of timing variance. Quarterly Journal of Experimental Psychology, 65, 1086-1100. doi: 10.1080/17470218.2011.640404

Taxt, T., Hjort, N. L., \& Eikvil, L. (1990). Statistical classification using a linear mixture of two multinormal probability densities. Pattern Recognition Letters, 12(12), 731-737. doi:10.1016/0167-8655(91) 90070-3

Whiteside, S. P., \& Lynam, D. R. (2000). The Five Factor Model and impulsivity: using a structural model of personality to understand impulsivity. Personality and Individual Differences, 30(4), 669689. doi:10.1016/S0191-8869(00)00064-7 